\title{
ON SOLUTIONS OF SOME NON-LINEAR DIFFERENTIAL EQUATIONS IN CONNECTION TO BRÜCK CONJECTURE
}

\author{
DILIP CHANDRA PRAMANIK AND MANAB BISWAS
}

\begin{abstract}
In this paper, we investigate on the non-constant entire solutions of some non-linear complex differential equations in connection to Brück conjecture and prove some results which improve and extend the results of Xu and Yang[Xu HY, Yang LZ. On a conjecture of R. Brück and some linear differential equations. Springer Plus 2015; 4:748,:1-10, DOI 10.1186/s40064-015-1530-5.]
\end{abstract}

\section{Introduction}

Let $f(z)$ be a nonconstant meromorphic function in the complex plane $\mathbb{C}$. We assume that the reader is familiar with the fundamental results and the standard notations of the Nevanlinna's value distribution theory such as $T(r, f), m(r, f), N(r, f)$ (e.g., see [2, 5, 12, 13]). By $S(r, f)$ we denote any quantity satisfying $S(r, f)=o(T(r, f))$ as $r \rightarrow+\infty$, possibly outside a set of $r$ with finite linear measure. A meromorphic function $\alpha(z)$ is said to be small with respect to $f(z)$ if $T(r, \alpha)=S(r, f)$.

Let $f(z)$ and $g(z)$ be two nonconstant meromorphic functions. For a small function $a(z)$ of $f$ and $g$, if $f(z)-a(z)$ and $g(z)-a(z)$ have same zeros with same multiplicities, we say that $f(z)$ and $g(z)$ share the function $a(z)$ CM (counting multiplicities) and if $f(z)-a(z)$ and $g(z)-a(z)$ have same zeros with ignoring multiplicities, we say that $f(z)$ and $g(z)$ share $a(z)$ IM (ignoring multiplicities). Note that $a(z)$ can be a value in $\mathbb{C} \cup\{\infty\}$.

For an entire function $f(z)=\sum_{n=0}^{\infty} a_{n} z^{n}$ the central index $v(r, f)$ is the greatest exponent $m$ such that $\left|a_{m}\right| r^{m}=\mu(r, f)$, where $\mu(r, f)=\max _{n \geq 0}\left|a_{n}\right| r^{n}$ denote the maximum term of $f$ on $|z|=r$. In this paper, we also need the following definitions.

Received May 18, 2017, accepted June 28, 2017.

2010 Mathematics Subject Classification. 39B32, 30D35.

Key words and phrases. Entire function, Nevanlinna Theory, differential equations in the complex domain.

Corresponding author: Dilip Chandra Pramanik. 
Definition 1.1. Let $f(z)$ be a nonconstant meromorphic function, then the order $\sigma(f)$ of $f(z)$ is defined by

$$
\sigma(f)=\limsup _{r \rightarrow+\infty} \frac{\log T(r, f)}{\log r}=\limsup _{r \rightarrow+\infty} \frac{\log \log M(r, f)}{\log r}
$$

and the lower order $\mu(f)$ of $f(z)$ is defined by

$$
\mu(f)=\liminf _{r \rightarrow+\infty} \frac{\log T(r, f)}{\log r}=\liminf _{r \rightarrow+\infty} \frac{\log \log M(r, f)}{\log r}
$$

where and in the sequel

$$
M(r, f)=\max _{|z|=r}|f(z)|
$$

Definition 1.2. [[5]] The type $\tau(f)$ of an entire function $f(z)$ with $0<\sigma(f)=\sigma<+\infty$ is defined by

$$
\tau(f)=\limsup _{r \rightarrow+\infty} \frac{\log M(r, f)}{r^{\sigma}} .
$$

Following Yi an Yang [13] we define

Definition 1.3. Let $f$ be a nonconstant meromorphic function, the hyper order $\sigma_{2}(f)$ of $f(z)$ is defined as follows

$$
\sigma_{2}(f)=\limsup _{r \rightarrow+\infty} \frac{\log \log T(r, f)}{\log r}
$$

and finally

Definition 1.4. Let $n_{0 j}, n_{1 j}, n_{2 j}, \ldots, n_{k j}$ are non negative integers. The expression $M_{j}[f]=$ $(f)^{n_{0} j}\left(f^{(1)}\right)^{n_{1 j}}\left(f^{(2)}\right)^{n_{2} j} \cdots\left(f^{(k)}\right)^{n_{k j}}$ is called a differential monomial generated by $f$ of degree $d\left(M_{j}\right)=\sum_{i=0}^{k} n_{i j}$ and weight $\Gamma_{M_{j}}=\sum_{i=0}^{k}(i+1) n_{i j}$. The sum $P[f]=\sum_{j=1}^{t} a_{j} M_{j}[f]$ is called a differential polynomial generated by $f$ of degree $\bar{d}(P)=\max \left\{d\left(M_{j}\right): 1 \leq j \leq t\right\}$ and weight $\Gamma_{P}=\max \left\{\Gamma_{M_{j}}: 1 \leq j \leq t\right\}$, where $a_{j} \neq 0(j=1,2, \ldots, t)$ and $T\left(r, a_{j}\right)=S(r, f)$ for $j=1,2, \ldots, t$. The numbers $\underline{d}_{P}=\min \left\{d\left(M_{j}\right): 1 \leq j \leq t\right\}$ and $k$ ( the highest order of the derivative of $f$ in $P[f]$ ) are called respectively the lower degree and the order of $P[f] . P[f]$ is said to be homogeneous if $\bar{d}_{P}=\underline{d}_{P} . P[f]$ is called a Linear Differential Polynomial generated by $f$ if $\bar{d}_{P}=1$. Otherwise, $P[f]$ is called Non-linear Differential Polynomial. We denote by $Q=\max \left\{\Gamma_{M_{j}}-d\left(M_{j}\right): 1 \leq j \leq t\right\}=\max \left\{\sum_{i=1}^{k} i . n_{i j}: 1 \leq j \leq t\right\}$

Rubel and Yang [10] proved that if a nonconstant entire function $f$ and its derivative $f^{\prime}$ share two distinct finite complex numbers CM, then $f=f^{\prime}$. How is the relation between $f$ and $f^{\prime}$, if an entire function $f$ and its derivative $f^{\prime}$ share one finite complex number $a$ CM? Brück [1] made the conjecture that if $f$ is a nonconstant entire function satisfying $\sigma_{2}(f)<\infty$, 
where $\sigma_{2}(f)$ is not a positive integer and if $f$ and $f^{\prime}$ share one finite complex number $a$ CM, then $f^{\prime}-a=c(f-a)$ for some nonzero finite complex number $c$. In 1998, Gundersen and Yang [4] proved that the conjecture is true for entire functions of finite order. Also, in 2008 $\mathrm{Li}$ and Cao[8] improved the Brück conjecture for entire function and its derivation sharing polynomials and proved the following theorem:

Theorem 1.1. Let $\phi(z)$ be any polynomial. If $f$ is a nonconstant entire solution of the equation $f^{(k)}-Q_{1}=e^{\phi}\left(f-Q_{2}\right)$, where $Q_{1}$ and $Q_{2}$ are non-zero polynomials, then $\sigma_{2}(f)=$ degree of $\phi$.

Mao [9] improve the above theorem in which he replaced the k-th derivative $f^{(k)}$ by the linear differential polynomial $L(f)=a_{k} f^{(k)}+a_{k-1} f^{(k-1)}+\cdots+a_{0} f$ and prove that

Theorem 1.2. Let $a_{k} \equiv 0, a_{k-1}, \ldots, a_{0}, P(z)$ be polynomials, $k \geq 1$ and $f$ be an entire function of order $\sigma(f)>\max _{0 \leq j \leq k-1}\left\{\frac{\operatorname{deg} a_{j}-\operatorname{deg} a_{k}}{k-j}, 0\right\}$ and hyper-order $\sigma_{2}(f)<\frac{1}{2}$. If $f$ and $L(f)$ share $P C M$, then $\frac{L(f)-P}{f-P}=c$ for a non zero constant $c$.

Later in 2009 Chang and Zhu [3] proved that Brück conjecture is true if the constant $a$ is replaced by a function $a(z)$, provided $\sigma(a)<\sigma(f)$.

Theorem 1.3. Let $f$ be an entire function of finite order and $a(z)$ be a function such that $\sigma(a)<$ $\sigma(f)<+\infty$. If $f$ and $f^{\prime}$ share $a(z) C M$, then $\frac{f^{\prime}-a}{f-a}=c$ for some constant $c \neq 0$.

In the year 2015, Xu and Yang [11] prove the following theorems:

Theorem 1.4. Let $f(z)$ and $\alpha(z)$ be two nonconstant entire functions and satisfy $0<\sigma(\alpha)=$ $\sigma(f)<+\infty, \tau(f)>\tau(\alpha)$ and let $\phi(z)$ be a polynomial such that

$$
\sigma(f)>\operatorname{deg}(\phi)+\max _{0 \leq j \leq k-1}\left\{\frac{\operatorname{deg} a_{j}-\operatorname{deg} a_{k}}{k-j}, 0\right\} .
$$

If $f$ is a nonconstant entire solution of the following differential equation $L(f)-\alpha(z)=(f(z)-$ $\alpha(z)) e^{\phi(z)}$, where $L(f)$ is defined as above. Then $\phi(z)$ is a constant.

Theorem 1.5. Let $f(z)$ and $\alpha(z)$ be two nonconstant entire functions satisfying $0<\sigma(\alpha)=$ $\sigma(f)<+\infty, \tau(f)>\tau(\alpha)$ and let $\phi(z)$ be a polynomial such that

$$
\sigma(f)>\operatorname{deg}(\phi)+\max _{0 \leq j \leq k-1}\left\{\frac{\operatorname{deg} a_{j}-\operatorname{deg} a_{k}}{k-j}, 0\right\} .
$$

If $f$ is a nonconstant entire solution of the following differential equation $L_{1}(f)-\alpha(z)=(f(z)-$ $\alpha(z)) e^{\phi(z)}$, where $L_{1}(f)=L(f)+\beta(z)$ and $\beta$ is an entire function satisfying $0<\sigma(\beta)=\sigma(f)<$ $+\infty, \tau(f)>\tau(\beta)$. Then $\phi(z)$ is a constant. 
Theorem 1.6. Let $f(z)$ and $\alpha(z)$ be two nonconstant entire functions satisfying $\sigma(\alpha)<\mu(f)$, and let $\phi(z)$ be a polynomial such that

$$
\sigma(f)>\operatorname{deg}(\phi)+\max _{0 \leq j \leq k-1}\left\{\frac{\operatorname{deg} a_{j}-\operatorname{deg} a_{k}}{k-j}, 0\right\} .
$$

If $f$ is a nonconstant entire solution of the following differential equation $L_{1}(f)-\alpha(z)=(f(z)-$ $\alpha(z)) e^{\phi(z)}$, where $L_{1}(f)=L(f)+\beta(z)$ and $\beta$ is an entire function satisfying $\sigma(\beta)<\mu(f)$. Then $\sigma_{2}(f)=\operatorname{deg} \phi(z)$.

In this paper, we improve and extend the results of Xu and Yang [11] in which we replaced the linear differential polynomial by the differential polynomial $P[f]$ and $f$ by $f^{\bar{d}_{P}}$ and proved the following theorems:

Theorem 1.7. Let $f(z)$ and $\alpha(z)$ be two nonconstant entire functions and satisfy $0<\sigma(\alpha)=$ $\sigma(f)<+\infty$ and $\tau(f)>\tau(\alpha)$. Also, let $\phi(z)$ be a polynomial. If $f$ is a nonconstant entire solution of the following differential equation

$$
P[f]-\alpha(z)=\left(f^{\bar{d}_{P}}-\alpha(z)\right) e^{\phi(z)},
$$

then $\phi(z)$ is a constant.

Theorem 1.8. Let $f(z)$ and $\alpha(z)$ be two nonconstant entire functions and satisfy $0<\sigma(\alpha)=$ $\sigma(f)<+\infty$ and $\tau(f)>\tau(\alpha)$. Also, let $\phi(z)$ be a polynomial. If $f$ is a nonconstant entire solution of the following differential equation

$$
P[f]+\beta(z)-\alpha(z)=\left(f^{\bar{d}_{P}}-\alpha(z)\right) e^{\phi(z)},
$$

where $\beta(z)$ is an entire function satisfying $0<\sigma(\beta)=\sigma(f)<+\infty$ and $\tau(f)>\tau(\beta)$. Then $\phi(z)$ is a constant.

Theorem 1.9. Let $f(z)$ and $\alpha(z)$ be two nonconstant entire functions satisfying $\sigma(\alpha)<\mu(f)$ and $\phi(z)$ be a polynomial. If $f$ is a nonconstant entire solution of the following differential equation

$$
P[f]+\beta(z)-\alpha(z)=\left(f^{\bar{d}_{P}}-\alpha(z)\right) e^{\phi(z)},
$$

where $\beta(z)$ is an entire function satisfying $\sigma(\beta)<\mu(f)$. Then $\sigma_{2}(f)=\operatorname{deg} \phi$.

\section{Preparatory lemmas}

In this section we state some lemmas needed in the sequel. 
Lemma 2.1 ([7]). Let $f(z)$ be a transcendental entire function, $v(r, f)$ be the central index of $f(z)$. Then there exists a set $E \subset(1,+\infty)$ with finite logarithmic measure, we choose $z$ satisfying $|z|=r \notin[0,1] \cup E$ and $|f(z)|=M(r, f)$, we get

$$
\frac{f^{j}(z)}{f(z)}=\left\{\frac{v(r, f)}{z}\right\}^{j}(1+o(1)), \text { for } j \in N .
$$

Lemma 2.2([6]). Let $f(z)$ be an entire function of finite order $\sigma(f)=\sigma<+\infty$ and let $v(r, f)$ be the central index of $f$. Then

$$
\limsup _{r \rightarrow+\infty} \frac{\log v(r, f)}{\log r}=\sigma(f)
$$

and if $f$ is a transcendental entire function of hyper order $\sigma_{2}(f)$, then

$$
\limsup _{r \rightarrow+\infty} \frac{\log \log v(r, f)}{\log r}=\sigma_{2}(f) .
$$

Lemma 2.3 ([9]). Let $f(z)$ be a transcendental entire function and let $E \subset[1,+\infty)$ be a set having finite logarithmic measure. Then there exists $\left\{z_{n}=r_{n} e^{i \theta_{n}}\right\}$ such that $\left|f\left(z_{n}\right)\right|=M\left(r_{n}, f\right)$, $\theta_{n} \in[0,2 \pi), \lim _{n \rightarrow+\infty} \theta_{n}=\theta_{0} \in[0,2 \pi), r_{n} \notin E$ and if $0<\sigma(f)<+\infty$, then for any given $\varepsilon>0$ and sufficiently large $r_{n}$,

$$
r_{n}^{\sigma(f)-\varepsilon}<v\left(r_{n}, f\right)<r_{n}^{\sigma(f)+\varepsilon}
$$

If $\sigma(f)=+\infty$, then for any given large $K>0$ and sufficiently large $r_{n}$,

$$
v\left(r_{n}, f\right)>r_{n}^{K}
$$

Lemma 2.4 ([7]). Let $\phi(z)=b_{n} z^{n}+b_{n-1} z^{n-1}+\cdots+b_{0}$ with $b_{n} \neq 0$ be a polynomial. Then for every $\varepsilon>0$, there exists $r_{0}>0$ such that for all $r=|z|>r_{0}$ the inequalities

$$
(1-\varepsilon)\left|b_{n}\right| r^{n} \leq|\phi(z)| \leq(1+\varepsilon)\left|b_{n}\right| r^{n}
$$

hold.

Lemma 2.5 ([11]). Let $f(z)$ and $A(z)$ be two entire functions with $0<\sigma(f)=\sigma(A)=\sigma<+\infty$, $0<\tau(A)=\tau(f)<+\infty$, then there exists a set $E \subset[1,+\infty)$ that has infinite logarithmic measure such that for all $r \in E$ and a positive number $\kappa$, we have

$$
\frac{M(r, A)}{M(r, f)}<\exp \left\{-\kappa r^{\sigma}\right\}
$$

\section{Proofs of the main results}

Proof of Theorem 1.7. Suppose that $\operatorname{deg} \phi=m \geq 1$. Let

$$
\phi(z)=b_{m} z^{m}+b_{m-1} z^{m-1}+\cdots+b_{0},
$$


where $b_{m}, b_{m-1}, \ldots, b_{0}$ are constants and $b_{m} \neq 0$. Thus, it follows from (1.1) and Lemma 2.4 that

$$
\begin{aligned}
\left|b_{m}\right| r^{m}(1+o(1)) & =|\phi(z)|=\left|\log \frac{\frac{P[f]}{f^{\bar{d}_{P}}}-\frac{\alpha}{f^{\bar{d}_{P}}}}{1-\frac{\alpha}{f^{\bar{d}_{P}}}}\right| \\
& =\left|\log \frac{\frac{P[f]}{f^{\bar{d}_{P}}}-\frac{\alpha}{f} \cdot \frac{1}{f^{\bar{d}_{P}-1}}}{1-\frac{\alpha}{f} \cdot \frac{1}{f^{\bar{d}_{P}-1}}}\right|
\end{aligned}
$$

Since for each $j=1,2, \ldots, t$,

$$
\begin{aligned}
M_{j}[f] & =(f)^{n_{0 j}}\left(f^{(1)}\right)^{n_{1 j}}\left(f^{(2)}\right)^{n_{2} j} \cdots\left(f^{(k)}\right)^{n_{k j}} \\
& =f^{\left(\sum_{i=0}^{k} n_{i j}\right)} \prod_{i=1}^{k}\left(\frac{f^{(i)}}{f}\right)^{n_{i j}} \\
& =f^{d_{M_{j}}} \prod_{i=1}^{k}\left(\frac{f^{(i)}}{f}\right)^{n_{i j}}
\end{aligned}
$$

and from Lemma 2.1, there exists a subset $E_{1} \subset(1,+\infty)$ with finite logarithmic measure, such that for some point $|z|=r e^{i \theta}(\theta \in[0,2 \pi]), r \notin E_{1}$ and $M(r, f)=|f(z)|$, we have

$$
\frac{f^{i}(z)}{f(z)}=\left\{\frac{v(r, f)}{z}\right\}^{i}(1+o(1)), 1 \leq i \leq k .
$$

Thus, it follows that

$$
\frac{M_{j}[f]}{f^{d_{M_{j}}}}=\prod_{i=1}^{k}\left\{\frac{v(r, f)}{z}\right\}^{i . n_{i j}}(1+o(1))=\left\{\frac{v(r, f)}{z}\right\}^{\left(\sum_{i=1}^{k} i . n_{i j}\right)}(1+o(1))
$$

and

$$
\begin{aligned}
\frac{P[f]}{f^{\bar{d}_{P}}} & \leq \sum_{j=1}^{t}\left|a_{j}\right|\left|\frac{M_{j}[f]}{f^{\bar{d}_{P}}}\right| \\
& \leq \sum_{j=1}^{t}\left|a_{j}\right|\left|\frac{M_{j}[f]}{f^{d_{M_{j}}}}\right| \\
& \leq \sum_{j=1}^{t}\left|a_{j}\right|\left|\frac{v(r, f)}{z}\right|^{\left(\sum_{i=1}^{k} i . n_{i j}\right)}(1+o(1)) \\
& \leq \sum_{j=1}^{t}\left|a_{j}\right|\left|\frac{v(r, f)}{z}\right|_{(1+o(1))}^{Q}
\end{aligned}
$$




$$
\leq\left|\frac{v(r, f)}{z}\right|^{Q} \cdot\left(\sum_{j=1}^{t}\left|a_{j}\right|\right) \cdot(1+o(1)) .
$$

From Lemma 2.3 there exists $\left\{z_{n}=r_{n} e^{i \theta_{n}}\right\}$ such that $\left|f\left(z_{n}\right)\right|=M\left(r_{n}, f\right), \theta_{n} \in[0,2 \pi]$, $\lim _{n \rightarrow \infty} \theta_{n}=\theta_{0} \in[0,2 \pi], r_{n} \notin E_{1}$, then for any given $\varepsilon>0$ and sufficiently large $r_{n}$,

$$
r_{n}^{\sigma(f)-\varepsilon}<v\left(r_{n}, f\right)<r_{n}^{\sigma(f)+\varepsilon}
$$

Then, from (3.2) and (3.3) we have

$$
\begin{aligned}
\frac{P[f]}{f^{\bar{d}_{P}}} & \leq\left\{\frac{v\left(r_{n}, f\right)}{r_{n}}\right\}^{Q} \cdot\left(\sum_{j=1}^{t}\left|a_{j}\right|\right) \cdot(1+o(1)) \\
& <r_{n}^{(\sigma(f)+\varepsilon-1) Q} \cdot\left(\sum_{j=1}^{t}\left|a_{j}\right|\right) \cdot(1+o(1)) .
\end{aligned}
$$

Since $0<\sigma(\alpha)=\sigma(f)<+\infty$ and $\tau(f)>\tau(\alpha)$, using Lemma 2.5, there exists a set $E \subset$ $[1,+\infty)$ that has infinite logarithmic measure such that for a sequence $\left\{r_{n}\right\}_{n=1}^{\infty} \in E_{2}=E-E_{1}$, we have

$$
\frac{M\left(r_{n}, \alpha\right)}{M\left(r_{n}, f\right)}<\exp \left\{-\kappa r_{n}^{\sigma(f)}\right\} \rightarrow 0 \text { as } n \rightarrow+\infty .
$$

From (3.1), (3.4) and (3.5) and Lemma 2.2, we get that

$$
\left|b_{m}\right| r^{m}(1+o(1))=|\phi(z)|=O\left(\log r_{n}\right),
$$

which is impossible. Thus, $\phi(z)$ is not a polynomial, that is, $\phi(z)$ is a constant.

Proof of Theorem 1.8. Rewritting (1.2) as

$$
\frac{\frac{P[f]}{f^{\bar{d}_{P}}}+\frac{\beta}{f} \cdot \frac{1}{f^{\bar{d}_{P}-1}}-\frac{\alpha}{f} \cdot \frac{1}{f^{\bar{d}_{P}-1}}}{1-\frac{\alpha}{f} \cdot \frac{1}{f^{\bar{d}_{P}-1}}}=e^{\phi(z)} .
$$

Our assumptions on $\tau$ and $\sigma$ values give, using Lemma 2.5, that there exists a set $E \subset[1,+\infty$ ) that has infinite logarithmic measure such that for a sequence $\left\{r_{n}\right\}_{n=1}^{\infty} \in E_{3}=E-E_{1}$, we have

$$
\frac{M\left(r_{n}, \alpha\right)}{M\left(r_{n}, f\right)}<\exp \left\{-\kappa r_{n}^{\sigma(f)}\right\} \rightarrow 0 \text { as } n \rightarrow+\infty
$$

and

$$
\frac{M\left(r_{n}, \beta\right)}{M\left(r_{n}, f\right)}<\exp \left\{-\kappa r_{n}^{\sigma(f)}\right\} \rightarrow 0 \text { as } n \rightarrow+\infty .
$$

Proceeding as in the proof of Theorem 1.7 we can show that $\phi(z)$ is a constant. 
Proof of Theorem 1.9. We will consider two cases (i) $\sigma(f)<+\infty$ and (ii) $\sigma(f)=+\infty$.

Case (i). Suppose that $\sigma(f)<+\infty$. Then $\sigma_{2}(f)=0$. Since $\sigma(\alpha)<\mu(f)$ and $\sigma(\beta)<\mu(f)$, from definitions of the order and the lower order, there exists infinite sequence $\left\{z_{n}\right\}_{n=1}^{\infty}$, we have

$$
\frac{\left|\alpha\left(z_{n}\right)\right|}{\left|f\left(z_{n}\right)\right|} \rightarrow 0 \text { and } \frac{\left|\beta\left(z_{n}\right)\right|}{\left|f\left(z_{n}\right)\right|} \rightarrow 0 \text { as } n \rightarrow \infty .
$$

Thus, by using the same argument as in Theorem 1.7, we get that $\phi(z)$ is a constant, that is, $\operatorname{deg} \phi=0$. Therefore, $\sigma_{2}(f)=\operatorname{deg} \phi$.

Case (ii). Suppose that $\sigma(f)=+\infty$.

Rewritting (1.3), we have

$$
\frac{\frac{P[f]}{f^{\bar{d}_{P}}}+\frac{\beta}{f} \cdot \frac{1}{f^{\bar{d}_{P}-1}}-\frac{\alpha}{f} \cdot \frac{1}{f^{\bar{d}_{P}-1}}}{1-\frac{\alpha}{f} \cdot \frac{1}{f^{\overline{d_{P}}-1}}}=e^{\phi(z)} .
$$

Since for each $j=1,2, \ldots, t$,

$$
\begin{aligned}
M_{j}[f] & =(f)^{n_{0 j}}\left(f^{(1)}\right)^{n_{1 j}}\left(f^{(2)}\right)^{n_{2} j} \cdots\left(f^{(k)}\right)^{n_{k j}} \\
& =f^{\left(\sum_{i=0}^{k} n_{i j}\right)} \prod_{i=1}^{k}\left(\frac{f^{(i)}}{f}\right)^{n_{i j}} \\
& =f^{d_{M_{j}}} \prod_{i=1}^{k}\left(\frac{f^{(i)}}{f}\right)^{n_{i j}}
\end{aligned}
$$

and from Lemma 2.1, there exists a subset $E_{4} \subset[1,+\infty)$ with finite logarithmic measure, we choose $z$ satisfying $|z|=r \notin[0,1] \cup E_{4}$ and $|f(z)|=M(r, f)$, we have

$$
\frac{f^{i}(z)}{f(z)}=\left\{\frac{v(r, f)}{z}\right\}^{i}(1+o(1)), 1 \leq i \leq k .
$$

Thus, it follows that

$$
\frac{M_{j}[f]}{f^{d_{M_{j}}}}=\prod_{i=1}^{k}\left\{\frac{v(r, f)}{z}\right\}^{i . n_{i j}}(1+o(1))=\left\{\frac{v(r, f)}{z}\right\}^{\left(\sum_{i=1}^{k} i . n_{i j}\right)}(1+o(1))
$$

and

$$
\begin{aligned}
\frac{P[f]}{f^{\bar{d}_{P}}} & \leq \sum_{j=1}^{t}\left|a_{j}\right|\left|\frac{M_{j}[f]}{f^{\bar{d}_{P}}}\right| \\
& \leq \sum_{j=1}^{t}\left|a_{j}\right| \frac{M_{j}[f]}{f^{d_{M_{j}}}} \mid
\end{aligned}
$$




$$
\begin{aligned}
& \leq \sum_{j=1}^{t}\left|a_{j}\right|\left|\frac{v(r, f)}{z}\right|^{\left(\sum_{i=1}^{k} i \cdot n_{i j}\right)}(1+o(1)) \\
& \leq \sum_{j=1}^{t}\left|a_{j}\right|\left|\frac{v(r, f)}{z}\right|^{Q}(1+o(1)) \\
& \leq\left|\frac{v(r, f)}{z}\right|^{Q} \cdot\left(\sum_{j=1}^{t}\left|a_{j}\right|\right) \cdot(1+o(1)) .
\end{aligned}
$$

Since $\sigma(f)=+\infty$, then it follows from Lemma 2.3 that there exists $\left\{z_{n}=r_{n} e^{i \theta_{n}}\right\}$ with $\left|f\left(z_{n}\right)\right|=M\left(r_{n}, f\right), \theta_{n} \in[0,2 \pi], \lim _{n \rightarrow \infty} \theta_{n}=\theta_{0} \in[0,2 \pi], r_{n} \notin E_{5} \subset[1,+\infty)$, such that for any large constant $K$ and for sufficiently large $r_{n}$, we have

$$
v\left(r_{n}, f\right) \geq r_{n}^{K}
$$

Since $\sigma(\alpha)<\mu(f)$ and $\sigma(\beta)<\mu(f)$, from definitions of order and lower order, there exists infinite sequence $\left\{z_{n}\right\}_{n=1}^{\infty}$, we have

$$
\frac{\left|\alpha\left(z_{n}\right)\right|}{\left|f\left(z_{n}\right)\right|} \rightarrow 0 \text { and } \frac{\left|\beta\left(z_{n}\right)\right|}{\left|f\left(z_{n}\right)\right|} \rightarrow 0 \text { as } n \rightarrow \infty .
$$

Thus, it follows from (1.3), (3.6), (3.7) and (3.8) that

$$
e^{\phi\left(z_{n}\right)} \leq\left\{\frac{v\left(r_{n}, f\right)}{r_{n}}\right\}^{Q} \cdot\left(\sum_{j=1}^{t}\left|a_{j}\right|\right) \cdot(1+o(1)) .
$$

Let

$$
\phi(z)=b_{m} z^{m}+b_{m-1} z^{m-1}+\cdots+b_{0},
$$

where $b_{m}, b_{m-1}, \ldots, b_{0}$ are constants and $b_{m} \neq 0, m \geq 1$. From Lemma 2.4, there exists sufficiently large positive number $r_{0}$ and $n_{0} \in N_{+}$, such that for sufficiently large positive integer $n>n_{0}$ satisfying $\left|z_{n}\right|=r_{n}>r_{0}$, we have for every $\varepsilon^{\prime}>0$

$$
\log \left|b_{m}\right|+m \log \left|z_{n}\right|+\log \left|1-\varepsilon^{\prime}\right| \leq \log \left|\phi\left(z_{n}\right)\right| \leq\left|\log \log e^{\phi\left(z_{n}\right)}\right| .
$$

It follows from (3.9) that

$$
\begin{aligned}
\left|\log \log e^{\phi\left(z_{n}\right)}\right| & \leq \log \log \left(\sum_{j=1}^{t}\left|a_{j}\right|\right)+\log \log v\left(r_{n}, f\right)+\log \log r_{n}+O(1) \\
& \leq \log \log v\left(r_{n}, f\right)+O\left(\log \log r_{n}\right)
\end{aligned}
$$

Thus, we have from (3.10) and (3.11) and Lemma 2.2 that

$$
m=\operatorname{deg} \phi \leq \sigma_{2}(f) .
$$


Also, it follows from (3.9) and Lemma 2.4 that

$$
M\left(r_{n}, e^{\phi\left(z_{n}\right)}\right) \geq\left\{\frac{v\left(r_{n}, f\right)}{r_{n}}\right\}^{Q} \cdot\left(\sum_{j=1}^{t}\left|a_{j}\right|\right) .
$$

Then, we have

$$
\left\{v\left(r_{n}, f\right)\right\}^{Q} \leq\left(\sum_{j=1}^{t}\left|a_{j}\right|\right)^{-1} \cdot\left(r_{n}\right)^{Q} \cdot M\left(r_{n}, e^{\phi\left(z_{n}\right)}\right) .
$$

Thus, it follows from (3.13) and Lemma 2.2 that

$$
\begin{aligned}
\sigma_{2}(f) & =\limsup _{r_{n} \rightarrow+\infty} \frac{\log \log v\left(r_{n}, f\right)}{\log r_{n}} \\
& =\limsup _{r_{n} \rightarrow+\infty} \frac{\log \log \left(v\left(r_{n}, f\right)\right)^{Q}}{\log r_{n}} \\
& \leq \limsup _{r_{n} \rightarrow+\infty} \frac{\log \log \left(\left(\sum_{j=1}^{t}\left|a_{j}\right|\right)^{-1} \cdot\left(r_{n}\right)^{Q} \cdot M\left(r_{n}, e^{\phi\left(z_{n}\right)}\right)\right)}{\log r_{n}} \\
& =\sigma\left(e^{\phi}\right) .
\end{aligned}
$$

Since $\phi(z)$ is a polynomial, then $\sigma\left(e^{\phi}\right)=\operatorname{deg} \phi=m$. By combining (3.12) and (3.14), we have $\sigma_{2}(f)=\operatorname{deg} \phi$.

Corollary 3.1. Let $f(z)$ and $\alpha(z)$ be two nonconstant entire functions and satisfy $\sigma(\alpha)<\mu(f)$. Also, let $\phi(z)$ be a polynomial. If $f$ is a nonconstant entire solution of the following differential equation

$$
P[f]-\alpha(z)=\left(f^{\bar{d}_{P}}-\alpha(z)\right) e^{\phi(z)},
$$

then $\sigma_{2}(f)=\operatorname{deg} \phi$.

\section{Acknowledgement}

The authors would like to thank Prof. Indrajit Lahiri for his suggestions.

\section{References}

[1] R. Brück, On entire functions which share one value CM with their first derivative, Results Math., 30 (1996), 21-24.

[2] W. Cherry and Z. Ye, Nevanlinnas Theory of Value Distribution. Springer-Verlag, 2001.

[3] J. M. Chang and Y. Z. Zhu, Entire functions that share a small function with their derivatives, J. Math. Anal. Appl., 351 (2009), 491-496. 
[4] G. G. Gundersen and L. Z. Yang, Entire functions that share one value with one or two of their derivatives, J Math Anal Appl, 223 (1998), 85-95.

[5] W. K. Hayman, Meromorphic Functions, The Clarendon Press, Oxford, 1964.

[6] Y. Z. He and X. Z. Xiao, Algebroid functions and ordinary differential equations, Science Press, Beijing, 1988.

[7] I. Laine, Nevanlinna Theory and Complex Differential Equations, de Gruyter, Berlin, 1993.

[8] X. M. Li and C. C. Cao, Entire functions sharing one polynomial with their derivatives, Proc. Indian Acad. Sci. Math. Sci., 118 (2008), 13-26.

[9] Z. Q. Mao, Uniqueness theorems on entire functions and their linear differential polynomials, Results Math., 55 (2009), 447-456.

[10] L. Rubel and C. C. Yang, Values shared by an entire function and its derivative. Complex Analysis, Lecture Notes in Math., 599 (1977) Springer-Verlag, Berlin, 101-103.

[11] H. Y. Xu, L. Z. Yang, On a conjecture of R. Brück and some linear differential equations. Springer Plus, 4:748 (2015), 1-10.

[12] L. Yang, Value Distribution Theory. Springer-Verlag, Berlin, 1993.

[13] H. X. Yi and C. C. Yang, Uniqueness Theory of Meromorphic Functions, Science Press, Beijing, 1995.

Department of Mathematics, University of North Bengal, Raja Rammohanpur, Dist-Darjeeling, PIN- 734013, West Bengal, India.

E-mail: dcpramanik.nbu2012@gmail.com

Barabilla High School, P.O. Haptiagach, Dist-Uttar Dinajpur, PIN- 733202, West Bengal, India.

E-mail: manab_biswas83@yahoo.com 